\title{
Interstellar and intergalactic dynamos
}

\author{
M. Hanasz, D. Woltanski and K. Kowalik \\ Centre for Astronomy, Nicolaus Copernicus University, \\ Faculty of Physics, Astronomy and Informatics, ul. Grudziadzka 5, PL-87-100 Torun, Poland, \\ ${ }^{*}$ E-mail: mhanasz@astri.uni.torun.pl
}

\begin{abstract}
We review recent developments of amplification models of galactic and intergalactic magnetic field. The most popular scenarios involve variety of physical mechanisms, including turbulence generation on a wide range of physical scales, effects of supernovae, buoyancy as well as the magnetorotational instability. Other models rely on galaxy interaction, which generate galactic and intergalactic magnetic fields during galaxy mergers. We present also global galacticscale numerical models of the Cosmic Ray (CR) driven dynamo, which was originally proposed by Parker (1992). We conduct a series of direct CR+MHD numerical simulations of the dynamics of the interstellar medium (ISM), composed of gas, magnetic fields and CR components. We take into account CRs accelerated in randomly distributed supernova (SN) remnants, and assume that SNe deposit small-scale, randomly oriented, dipolar magnetic fields into the ISM. The amplification timescale of the large-scale magnetic field resulting from the CR-driven dynamo is comparable to the galactic rotation period. The process efficiently converts small-scale magnetic fields of SN-remnants into galactic-scale magnetic fields. The resulting magnetic field structure resembles the $\mathrm{X}$-shaped magnetic fields observed in edge-on galaxies.
\end{abstract}

Keywords. Galaxies: ISM, Magnetic Fields; ISM: Cosmic Rays, Magnetic Fields; MHD: Dynamos

\section{Introduction}

Galactic magnetic fields are observed with the aid of various techniques, such as radioobservations of continues emission from the interstellar medium (ISM), Zeemann observations of magnetic fields in molecular clouds and optical observations of polarized light scattered by interstellar dust particles (see the recent review by Beck (2012)).

It is commonly accepted that contemporary magnetic fields in galaxies have been produced by some kind of dynamo process. Dynamos require seed magnetic fields resulting from other physical processes. Various mechanisms operating prior to galaxy formation offer rather week magnetic fields of the strengths $\leqslant 10^{-19} \mathrm{G}$ (see the review by Widrow (2002) and references therein). Biermann battery acting during galaxy formation, can generate magnetic fields of the order of $10^{-21} \mathrm{G}$ on the scale of $10-20 \mathrm{kpc}$, that can be further compressed by $2-3$ orders of magnitude during the galactic collapse, leading to a $10^{-17} \mathrm{G}$ at the fully formed galaxy (Lesch and Chiba, 1995). Another possibility is that first generation of stars provided the initial galactic magnetic fields of the mean strength of $10^{-9} \mathrm{G}$ on galactic scales (Syrovatskii, 1970; Bisnovatyi-Kogan et al., 1973). Magnetic fields of the same order are expected from first AGNs (Rees, 2006).

Two types of dynamo are considered in the context of galactic and extragalactic magnetic fields: (1.) large-scale (mean field) dynamos, producing coherent magnetic fields on length-scales much larger than the scales of the energy-carrying turbulent eddies, and (2.) small-scale (fluctuation) dynamos resulting in amplification of magnetic fields on timescales of the order of eddy turnover time and length-scales smaller than the scales of energy carrying eddies. Magnetic fields appear coherent over scales of several $\mathrm{kpc}$ in contemporary galaxies, therefore large-scale dynamos provide a plausible amplification 
mechanism in disk galaxies. On the other hand, random magnetic fields are observed in galaxy clusters, indicating a possible action of small-scale dynamos.

\section{Mean field galactic dynamos.}

The mean field dynamo theory (Krause and Raedler, 1980) relies on splitting of magnetic field and velocity field into mean and fluctuating parts. The dynamo equation results from averaging of the induction equation, under the assumption of turbulence isotropy and scale separation in space and time. Application of the dynamo theory to galactic disks has been widely discussed in several reviews (Beck et al., 1996; Shukurov, 2004; Kulsrud and Zweibel, 2008).

In the local, thin disk approximation and under the assumption of axial symmetry (Ruzmaikin et al., 1988; Shukurov, 2004), the dynamo equation can be reduced to the following set of equations

$$
\begin{gathered}
\frac{\partial \overline{B_{r}}}{\partial t}=\frac{\partial}{\partial z}\left(\alpha \overline{B_{\varphi}}\right)+\beta \frac{\partial^{2} \overline{B_{r}}}{\partial z^{2}} \\
\frac{\partial \overline{B_{\varphi}}}{\partial t}=G \overline{B_{r}}+\beta \frac{\partial^{2} \overline{B_{r}}}{\partial z^{2}}
\end{gathered}
$$

describing amplification of the poloidal and toroidal magnetic field components in terms of the parameters $G=r \frac{d \Omega}{d r}$ - the measure of differential rotation, $\alpha$ - the mean helicity, and $\eta_{t}$ - the turbulent diffusivity.

Substitution of turbulent length scales and velocities typical for ISM of disk galaxies $L_{t} \sim 100 \mathrm{pc}, V_{t} \sim c_{s} \sim 10 \mathrm{~km} \mathrm{~s}^{-1}$, and a typical vertical scale height of galactic disks $h \simeq 500 \mathrm{pc}$, together with the rotational shear parameter $G \simeq \Omega \simeq 10^{-15} \mathrm{~s}^{-1}$, allows one to make simple estimations of the turbulent transport coefficients $\alpha \sim 1 / 3 L_{t} \Omega \sim$ $1 \mathrm{~km} \mathrm{~s}^{-1}, \beta \sim 1 / 3 V_{t} L_{t} \sim 10^{26} \mathrm{~cm}^{2} s^{-1}$. With these parameters the dynamo equation admits exponentially growing solutions: $\bar{B} \propto \exp \left(t / t_{\mathrm{dyn}}\right)$, where $t_{\mathrm{dyn}} \sim(0.5 \div 1) \times 10^{9} \mathrm{yr}$ is the exponential growth time of the modes of the mean-field galactic dynamo. 5 Basic solutions of the linear system can be classified according to their reflection symmetry (dipolar and quadrupolar) with respect to the galactic midplane, and according to their azimuthal wavenumber $m=0,1,2, \ldots$. The most easily excited are the axisymmetricspiral (ASS, $m=0$ ) and bisymmetric-spiral (BSS, $m=1$ ) modes.

There are other useful approximations that allow to investigate the effects of spiral arms with analytical, semi-analytic or numeral methods, such as the 'no-z' approximation reducing the problem to the $(r, \varphi)$-plane, by the replacement of $\partial / \partial z$ by $1 / h$ in the dynamo equation (Shukurov, 2004). The standard numerical methods of fluid dynamics make it possible to construct 3-dimensional solutions of the dynamo equation (Gissinger et al., 2009).

Linear dynamo models neglect by definition the back-reaction of magnetic field on the dynamo transport coefficients. Vainshtein \& Cattaneo (1992) noted that, due to the rapidly growing small-scale, magnetic field $\boldsymbol{b}$, Lorentz force can make the large-scale dynamo inefficient

$$
|\overline{\boldsymbol{B}}|^{2} \simeq R_{m}^{-1} \boldsymbol{b}^{2}
$$

where the magnetic Reynolds number $R_{m}>>1$.

It has been noted that the catastrophic quenching of dynamo may be a consequence of the conservation of magnetic helicity in a medium of high electric conductivity. Blackman \& Field (2000) suggested that the losses of the small-scale magnetic helicity through the boundaries of the dynamo region can be essential for mean-field dynamo action (see the 
review by Brandenburg \& Subramanian (2005)) Conservation of magnetic helicity implies that generation of large-scale helical magnetic fields is associated with generation of an equal amount, but opposite-sign helicity at small scales.

The transport equation for small-scale helicity density $\chi$ derived from the helicity conservation law (see eg. Shukurov et al.(2006)) reads

$$
\frac{\partial \chi}{\partial t}+\nabla \cdot \boldsymbol{F}=-2 \mathcal{E} \cdot \overline{\boldsymbol{B}}-2 \eta \overline{\boldsymbol{j} \cdot \boldsymbol{b}}
$$

where $\boldsymbol{F}$ is the small-scale helicity flux, $\boldsymbol{j}=\boldsymbol{\nabla} \times \boldsymbol{b}$ is the electric current density and $\mathcal{E}$ is the mean electromotive force of turbulence. The helicity transport equation implies that magnetic helicity can be reduced by magnetic helicity flux, resulting from eg. advection by galactic winds, or dissipated by Ohmic resistivity.

Shukurov et al. (2006)) assumed helicity flux $\boldsymbol{F}=\chi \overline{\boldsymbol{U}}_{z}$, where $\overline{\boldsymbol{U}}_{z}$ proportional to the mean vertical speed of galactic wind, and then used the small-scale helicity equation to constrain the dependence of $\alpha(B)$. By the assumption that $\alpha=\alpha_{K}+\alpha_{m}$, where $\alpha_{K}=-\frac{1}{3} \tau \boldsymbol{u} \cdot \nabla \times \boldsymbol{u}$ and $\alpha_{m}$ is the magnetic part of the $\alpha$-effect, they demonstrated that $\alpha$ is reduced when $B^{2}$ approaches $B_{e q}^{2}=\rho \overline{u^{2}}$. The evolution equation for $\alpha_{m}$, resulting from the small-scale helicity equation, has been solved together with the mean-field dynamo equation for $B_{r}$ and $B_{\varphi}$. The solutions of the mean-field dynamo equation depends essentially on the vertical wind speed $\overline{\boldsymbol{U}}_{z}$. In absence of the vertical flow magnetic field amplification saturates at the level of magnetic fields weaker by orders of magnitude than the equilibrium value $B_{e q} \simeq 5 \mu \mathrm{G}$. It turns out that the helicity transport corresponding to the relatively small wind velocity of the order of $1 \mathrm{~km} \mathrm{~s}^{-1}$ leads to the growth of magnetic field up to $1 \mu \mathrm{G}$ values (weaker than the equilibrium value, but still significantly strong magnetic field), while excessive advection removes the mean field from the dynamo active region. Shukurov et al.(2006)) show that the vertical advection of small scale magnetic fields helps dynamo to avoid the catastrophic quenching, and demonstrate the importance of galactic winds and fountains for the efficiency of mean field dynamos.

\section{Magnetic field amplification scenarios in galaxies and clusters of galaxies}

\subsection{Magnetic field amplification in evolving galaxies}

Relying on the dynamo theory Arshakian et al.(2009) construct a three-phase model of magnetic field amplification in evolving galaxies. Their model, based on simple analytical estimations, starts with the Biermann battery in the first phase, providing seed magnetic fields of $10^{-18} \mathrm{G}$ during the epoch of dark matter halo formation. They propose that, in the second phase starting at cosmological redshifts $z \sim 20$, turbulence generated by thermal virialization in protogalactic halos, have driven the small-scale turbulent dynamo and amplified the seed field to the equipartition level within a few $10^{8} \mathrm{yr}$. In the third phase, starting from $z \sim 10$, large-scale magnetic fields were generated by large-scale dynamos on a timescale of several $10^{8} \mathrm{yr}$, from the small scale fields amplified in the second phase. According to this model, regular fields of $\mu \mathrm{G}$ strength and a few $\mathrm{kpc}$ coherence length were generated within 2 Gyr (at $z \simeq 3$ ) in galaxies similar to the Milky Way, but field-ordering on the coherence scale of the galaxy size required an additional 6 Gyr $($ at $z \simeq 0.5)$.

\subsection{Amplification of galactic magnetic fields during galaxy formation}

Wang \& Abel (2009) investigated magnetic field amplification during the gravitational collapse of a protogalactic cloud. They imposed a uniform seed field of $10^{-9} \mathrm{G}$ and found 
growth of the field to $\mu \mathrm{G}$ level in $\sim 500 \mathrm{Myr}$, during the disk formation phase. The efolding time of magnetic energy before saturation in this experiment was $\sim 50 \mathrm{Myr}$. They find that after saturation, the toroidal field in the disk dominates over the vertical component, while in the magnetized halo, the vertical component dominates over the toroidal component. Similarly, Beck et al. (2012) find efficient magnetic field amplification during the formation phase of a spherically symmetric galactic halo. They assume primordial seed field $10^{-18} \mathrm{G}$ and observe agglomeration of magnetic fields together with the gas within filaments and protohaloes, where it was amplified within a couple of hundred million years up to equipartition with the turbulent energy. The turbulence was generated by the gravitational collapse and by supernova feedback. Magnetic field strengths of $\simeq 10^{-6} \mathrm{G}$ are reached in the centre of the halo and drop to $\simeq 10^{-9} \mathrm{G}$ in the intergalactic medium. It has been shown that the magnetic field is amplified by turbulent small-scale dynamo action.

\subsection{Magnetic field amplification by galactic interactions}

Kotarba et al.(2011) studied magnetic field evolution by means of SPH/N-body simulations of galaxy collisions. They took into account various physical processes such as SN feedback, radiative cooling, star formation. The initial setup consisted of two, three, and more galaxies, whose dynamical parameters were adopted to permit subsequent mergers. By assumption of initial magnetic fields in the range $10^{-9}-10^{-6} \mu \mathrm{G}$ in the interstellar medium (ISM) and $10^{-12}-10^{-9} \mu \mathrm{G}$ in the intergalactic medium (IGM), they found the growth of magnetic fields up to the values of $1 \mu \mathrm{G}$ within the galaxies and $10^{-2} \mu \mathrm{G}$ in the IGM. Therefore, they find that galactic interactions are efficient drivers of magnetic field amplification. The final magnetic field strengths were independent from the assumed initial values. Geng et al.(2012) investigate amplification of magnetic fields in galaxy minor mergers for different galaxy mass ratios. They find that the magnetic field growth in merger remnants saturates at a common value of several $\mu \mathrm{G}$, and that higher impact energies of progenitor galaxies lead to a more efficient magnetic field amplification. Within the CDM hierarchical structure formation model all galaxies build up through several major and minor mergers. They conclude that the magnetization of the universe should be a natural part of galaxy formation and evolution.

\subsection{Magnetic field amplification in galaxy clusters}

Observational data indicate that intergalactic gas in clusters of galaxies is magnetized (see the reviews by Govoni \& Feretti (2004) and Ensslin et al. (2005)). Magnetic fields in galaxy clusters are detected primarily by measurements of Faraday rotation of the polarized emission from background radio galaxies, although synchrotron emission from some relatively small number of clusters is detected as well. Observations show that cluster fields are at the $\mu \mathrm{G}$ level, with values up to tens $\mu \mathrm{G}$ at the center of cooling core clusters. Cluster magnetic fields are likely to fluctuate over a wide range of spatial scales with values from a few kpc up to hundreds kpc.

The possible sources of seed magnetic fields can be active galaxies within clusters (Kronberg, 1994), galactic winds (Donnert et al., 2009; Dubois and Teyssier, 2010), galaxy mergers as well as magnetic fields striped from galaxies (Otmianowska-Mazur and Vollmer, 2003) due to their aerodynamic interaction with the intracluster medium.

Dolag et al. (2002) performed SPH MHD simulations of magnetic field growth in galaxy clusters in standard and open CDM cosmologies, varying the strength and the initial magnetic field configuration. They find that starting with magnetic fields of the order $10^{-9} \mathrm{G}$ at an initial redshift $z=15$ (20 for $\left.\Lambda \mathrm{CDM}\right)$, final fields reach $\mu \mathrm{G}$ strength at redshift $z=0$. The amplification factor $10^{3}$ exceeds the amplification factor from 
spherical collapse by an order of magnitude. The correlation lengths of the field is of the order $50-100 h^{-1} \mathrm{kpc}$. The field strengths follows the cluster density profiles outside the cluster core radii. The magnetic filed strength grows roughly exponentially with redshift: $|B(z)| \sim 10^{-2.5 z} \mu \mathrm{G}$.

Subramanian et al. (2006) argue, on the base of analytical estimations and numerical simulations, that random seed magnetic fields in galaxy clusters can be amplified by the small scale MHD dynamo to the strength of $2 \mu \mathrm{G}$ in $5 \mathrm{Gyr}$. The corresponding amplification factor of magnetic field is $10^{4}$, and the e-folding time is about $0.5 \mathrm{Gyr}$. They identify three distinct regimes of magnetic field amplification in clusters: (1.) fluctuation dynamo associated with turbulent flows with $v_{r m s}=300 \mathrm{~km} \mathrm{~s}^{-1}$ at scales of $200 \mathrm{pc}$, produces a $\mu \mathrm{G}$ random magnetic field, on scales $20-30 \mathrm{kpc}$, during the epoch of cluster formation and major mergers, (2.) a power-law decay of magnetic fields by a factor of 2 associated with simultaneous growth of their lengths scales by the same factor, and (3.) generation of $\sim 2 \mu \mathrm{G}$ magnetic fields by turbulent wakes of sub-clusters and galaxies in the cluster. Another option is that intergalactic magnetic fields have been striped from galaxies, however the magnetic field strength does not exceed $\simeq 0.1 \mu \mathrm{G}$, on scales of $1-30 \mathrm{kpc}$.

More recently Bonafede et al.(Bonafede et al., 2011) performed resistive MHD SPH simulations of magnetic field generation during formation of clusters during cosmological structure formation. They assumed initial uniform magnetic field $10^{-12} \mathrm{G}$, and performed simulations for a range of different values of magnetic diffusivity. They obtain magnetic field strength $\sim 1 \mu \mathrm{G}$ on average within the cluster, and up to $10 \mu \mathrm{G}$ in cluster cores.

It has been noted recently that the standard MHD approach may be inapplicable for cluster environments. The collisionless-MHD approach seems to provide a more appropriate formalism (see Dal Pino \& Santos-Lima, this Proceedings), although the standard collisional MHD-model provides reasonable results.

\subsection{Galactic dynamo due to supernova-driven interstellar turbulence}

Mac Low and Klessen (2004) have shown that supernovae are the dominant source of turbulence energy in the ISM. This result indicates that supernova activity is the main driver of the turbulent dynamo in Galaxies. Results of radio-continuum observations (Chyży, 2008) provide evidence for the correlation between the strength of turbulent components of interstellar magnetic fields and the star formation rate (SFR). On the other hand the FIR-radio correlation for a sample of galaxies (Bell, 2003) clearly shows that the total (mostly turbulent) galactic magnetic fields and star formation are connected (Beck, 2012). These facts provide evidence for the importance of star formation activity for generation of galactic magnetic fields. In the following we shall therefore focus on magnetic field generation mechanisms, connected to supernova rate, which is proportional to SFR.

Gressel et al. (2008b; 2008a; 2011) performed direct numerical simulations of a local 3D patch of interstellar medium. They took into account vertical stratification of the disk, sheared galactic rotation, effects of heating, cooling and heat conduction, focusing on the role of energy injection by supernova explosions. They observe exponential amplification of the regular magnetic field, on the timescale of $250 \mathrm{Myr}$, although the angular velocity of galactic rotation had to be enlarged artificially by a factor of $\sim 2$ above the real value to obtain magnetic field amplification effect. These authors derive dynamo parameters ( $\alpha, \eta$ tensors) for the simulated ISM, with the aid of test-field method. They find that the simulation results are consistent with the classical understanding of dynamo. 


\subsection{Magnetic field amplification by magnetorotational instability}

Magnetorotational instability has been recognized as the mechanism driving turbulence in accretion disks (Balbus and Hawley, 1998). Sellwood \& Balbus (1999) proposed that the magneto-rotational instability (MRI) may serve as the source of turbulence required for dynamo action in the outer disk, where the star-formation activity is low. Dziourkevitch et al. (2004) perform global 3D nonlinear MHD simulations of MRI in galactic disks. They find that the action of MRI leads to the generation toroidal and poloidal components of the mean magnetic fields in galactic disks. They suggest the possibility that magnetic fields of a few $\mu \mathrm{G}$ could have been produced in young galaxies within a few $10^{8}$ years.

\section{Cosmic-Ray-driven dynamo}

\subsection{Cosmic rays in the ISM}

The dynamical role of CRs was first recognized by Parker (1966), who noticed that a vertically stratified ISM which consists of thermal gas, magnetic fields and CRs is unstable due to buoyancy of the weightless components, i.e. the magnetic fields and the CRs. According to diffusive shock acceleration models CRs are continuously supplied to the ISM by SN remnants. Therefore, the buoyancy effects caused by CRs are expected in all star forming galaxies. Theories of diffusive shock acceleration predict that about $10 \%$ of the $\sim 10^{51}$ erg of the SN II explosion energy is converted to CR energy. Observational data indicate that gas, magnetic fields and CRs appear in approximate energetic equipartition, which means that all three components are dynamically coupled. In order to incorporate the CR propagation in MHD considerations we use the diffusion-advection equation (e.g. Schlickeiser \& Lerche (1985)) and take into account the CR pressure gradient in the gas equation of motion (see e.g. Berezinski et al. (1990)).

\subsection{Model assumptions}

The CR-driven dynamo was originally proposed by Parker (1992). Our model of the CR-driven dynamo involves the following elements (Hanasz et al., 2004; Hanasz et al., 2006; Hanasz et al., 2009a; Hanasz et al., 2009b): (1) The CR nuclear component described by the diffusion-advection transport equation, supplemented to the standard set of resistive MHD equations (Hanasz and Lesch, 2003). (2) CRs supplied in SN remnants. The CR input of individual SNe is assumed to be $10 \%$ of the typical SN kinetic energy output $\left(=10^{51} \mathrm{erg}\right)$, while the thermal energy output from supernovae is neglected. (3) Anisotropic CR diffusion along magnetic field lines (Giacalone and Jokipii, 1999), (4) Finite resistivity of the ISM in order to permit topological evolution of the galactic magnetic fields via anomalous resistivity processes (Hanasz et al., 2002), and/or via turbulent reconnection (Kowal et al., 2009) on small spatial scales, which are unresolved in our simulations. (5) An initial gas distribution in the disk which follows the model of the ISM in the Milky Way by Ferrière (1998). (6) Differential rotation of the interstellar gas, which currently follows an assumed form of a galactic gravitational potential.

We briefly mention that various properties of the shearing-box models of the CR-driven dynamo were discussed in a series of papers. Computations of the dynamo coefficients in Parker unstable disks with CRs and shear are described by Kowal et al. (2006) and by Otmianowska-Mazur et al. (2007). Synthetic radio-maps of a global galactic disk based on local CR-driven dynamo models exhibiting X-type structures were presented by Otmianowska-Mazur et al. (Otmianowska-Mazur et al., 2009). More recently, Siejkowski et al. (2010) demonstrated that the CR-driven dynamo can also work given the physical 

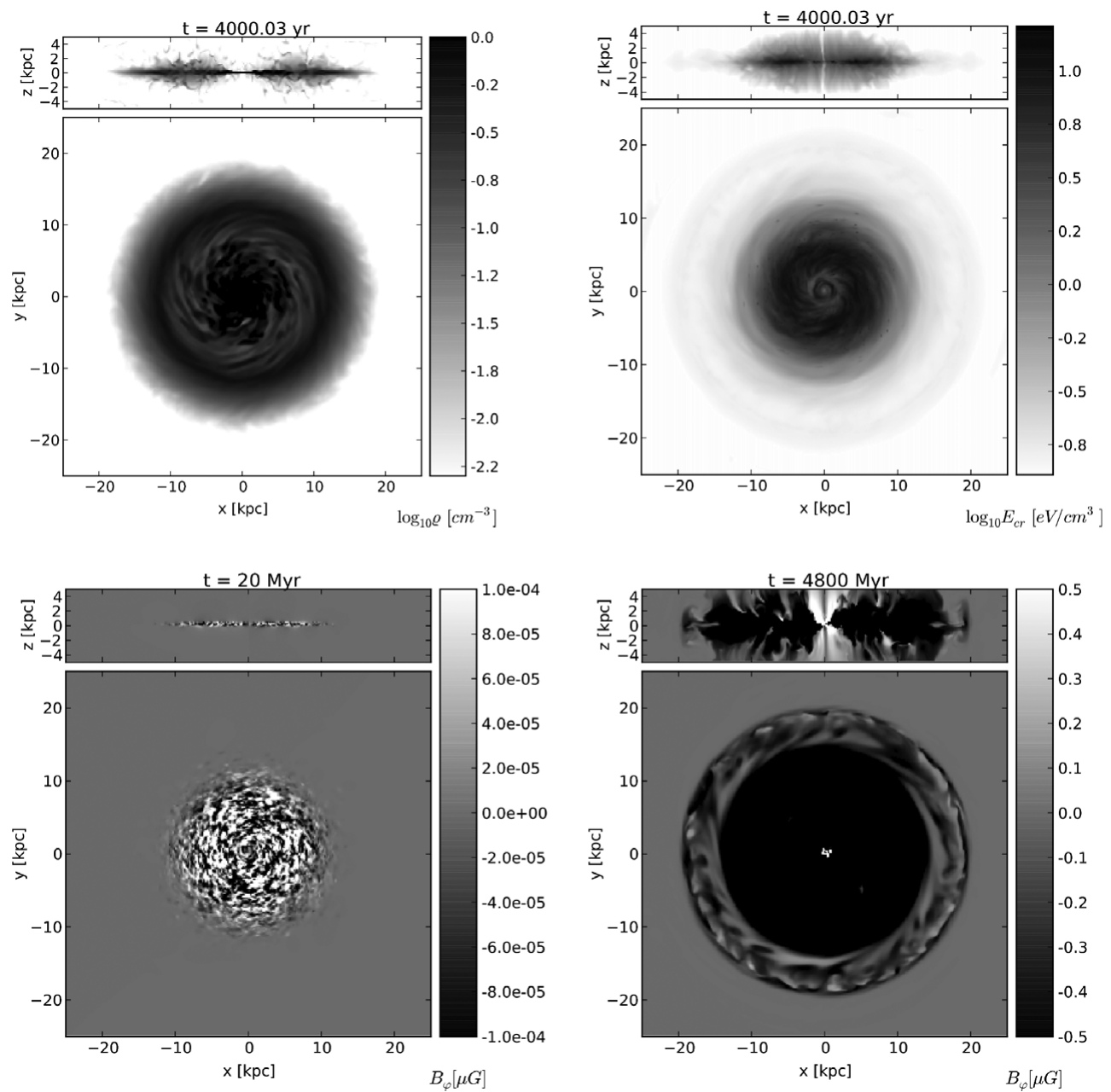

Figure 1. Top panels: logarithm of gas number density (left) and cosmic ray energy density (right) at $t=4 \mathrm{Gyr}$. Bottom panels: the distribution of toroidal magnetic field at $t=20 \mathrm{Myr}$ (left) and $t=4.8 \mathrm{Gyr}$ (right). Unmagnetized regions of the volume are grey, while positive and negative toroidal magnetic fields are marked lighter and darker, respectively. Note that the grey-scale scale in magnetic field maps is saturated to enhance weaker magnetic field structures in disk peripheries.

conditions of irregular galaxies, characterized by a relatively weak rotation and shearing rate.

\subsection{Global CR-driven dynamo simulations}

Our first realization of a global $\mathrm{CR}+\mathrm{MHD}$ galactic disk model relies on the following assumptions: First of all, we adopt analytical formulae for the gravitational potential corresponding to a system consisting of a galactic halo, bulge and disk (Allen and Santillan, 1991). We assume no magnetic field at $t=0$, and that weak $\left(10^{-4} \mu \mathrm{G}\right)$, dipolar, small scale $(r \sim 50 \mathrm{pc})$, randomly oriented magnetic fields are supplied locally in $10 \%$ of the SN remnants for $t \leqslant 1$ Gyr. We assume also that the SN rate is proportional to the star formation rate (SFR) which, on the other hand, is proportional to the initial gas column density. 


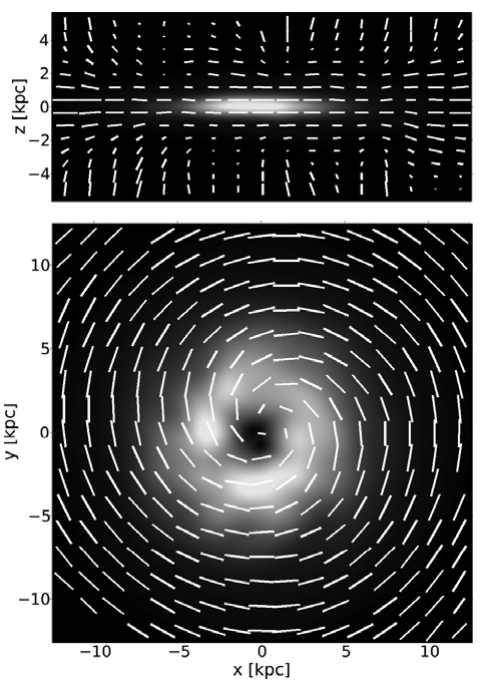

Figure 2. Synthetic radio maps of polarized intensity (PI) of synchrotron emission, together with polarization vectors are shown for the edge-on and face-on views of the galaxy at $t=4.8 \mathrm{Myr}$. Vectors direction resembles electric vectors rotated by $90^{\circ}$, and their lengths are proportional to the degree of polarization.

We find magnetic field amplification originating from the small-scale, randomly oriented dipolar magnetic fields, which is apparent through the exponential growth by several orders of magnitude of both the magnetic flux and the magnetic energy (details see Hanasz et al. (2009b)). The growth phase of the magnetic field starts at the beginning of the simulation. The growth of the magnetic field strength saturates at about $t=4 \mathrm{Gyr}$, reaching values of $3-5 \mu \mathrm{G}$ in the disk. During the amplification phase, magnetic flux and total magnetic energy grow by about 6 and 10 orders of magnitude, respectively. The average e-folding time of magnetic flux amplification is approximately equal to $270 \mathrm{Myr}$, corresponding to the rotation at the galactocentric radius $(\approx 10 \mathrm{kpc})$. The magnetic field is initially entirely random $(t=20 \mathrm{Myr})$, since it originates from randomly oriented magnetic dipoles. Later on, the toroidal magnetic field component forms a spiral structure revealing reversals in the plane of the disk. The magnetic field structure evolves gradually towards larger and lager scales. The toroidal magnetic field component becomes almost uniform inside the disk at $t=2.5 \mathrm{Gyr}$. The volume occupied by the well-ordered magnetic field expands continuously until the end of the simulation.

In order to visualize the magnetic field structure in a manner resembling radio observations of external galaxies, we construct synthetic radio maps of the synchrotron radio-emission, assuming that energy density of CR electrons equals 1\% energy density of CR nucleons. We apply standard procedures of line-of-sight integration of the stokes parameters $I, Q$, and $U$ for the polarized synchrotron emissivity. We neglect the effects of Faraday rotation.

In Figure 2, we show the polarized intensity of synchrotron emission (grey-scale maps), together with polarization vectors. Electric vectors, computed on the basis of integrated Stokes parameters, are rotated by $90^{\circ}$ to reproduce the magnetic field direction averaged along the line-of-sight, assuming vanishing Faraday rotation effects. The polarization vectors, indicating the mean magnetic field direction, reveal a regular spiral structure in the face-on view, and the so-called $X$-shaped structure in the edge-on view. A particular similarity can be noticed between our edge-on synthetic radio map and the radio maps of observed edge-on galaxies such as NGC 891 (Krause, 2009). In the present global model, 

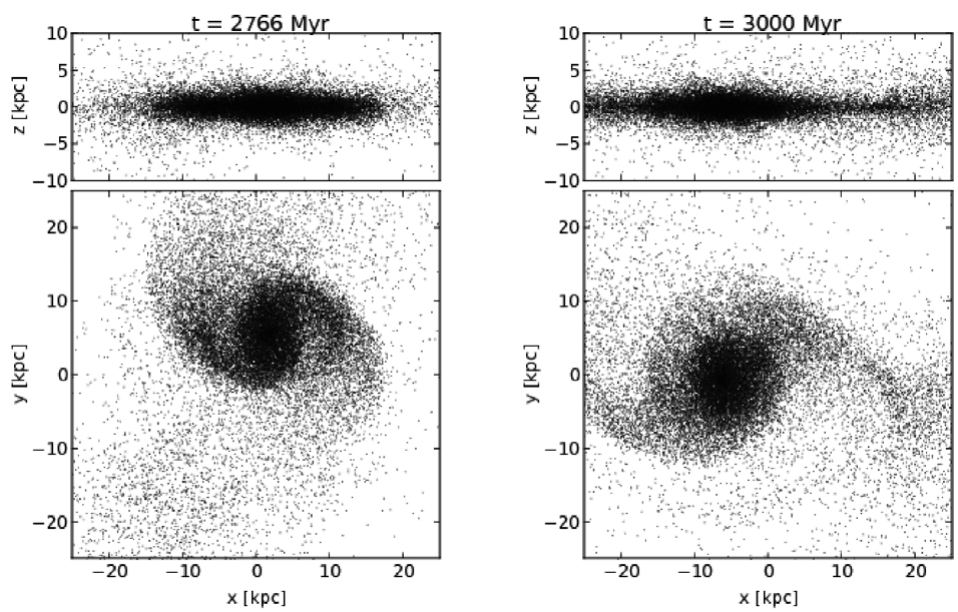

Figure 3. Two states of the N-body galactic disk prior to the galactic merger. The companion galaxy is apparent in the right panels.

the X-shaped configuration is an intrinsic property of the magnetic field structure, since it corresponds closely to the flaring radial distribution of magnetic field in the disk and its neighborhood, as shown in Figure 1.

The face-on synchrotron radio map reveals a spiral structure of the magnetic field, however, due to the assumed axisymmetric gravitational potential no features resembling spiral magnetic arms are present. To make the model more realistic, we incorporate non-axisymmetry in the gravitational potential, applying two different approaches. The first approach relies on the addition of an analytical elliptical perturbation to the axisymmetric gravitational potential (Kulpa-Dybeł et al., 2011). In presence of the bar perturbation the CR-driven dynamo reveals new properties, such as the presence of a ring-like structure as well as a shift of the magnetic arms with respect to the crests of spiral density waves.

As a further step towards more realistic galactic magnetic field models we perform N-body simulations of a disk-bulge-halo system (Wóltański et al., 2013), and interpolate the resulting gravitational potential onto the computational grid. We use this potential to compute the gravitational acceleration acting on the fluid components. In order to excite density waves in the galactic disk we add a small satellite galaxy, which ultimately merges with the main galaxy at $t=3.2 \mathrm{Gyr}$. Two snapshots of the N-body disk simulation are displayed in Fig. 3. The N-body part of the computation is performed with the VINE code (Wetzstein et al., 2009), and the CR+MHD part with the PIERNIK code. Fig. 4 shows the gas density and the toroidal magnetic field component. Similar to the case of an axisymmetric gravitational potential we observe efficient magnetic field amplification by the CR-driven dynamo. In the presence of spiral arms in the stellar and gas components the magnetic field also reveals a spiral structure. Moreover, we notice that both polarities of the azimuthal magnetic field are present at an advanced stage of magnetic field evolution. It appears that during the merger phase and afterwards the magnetic field structure becomes even more disordered, providing a possible explanation for the less regular magnetic field structures observed in interacting galaxies such as M51 (Fletcher et al., 2011). 

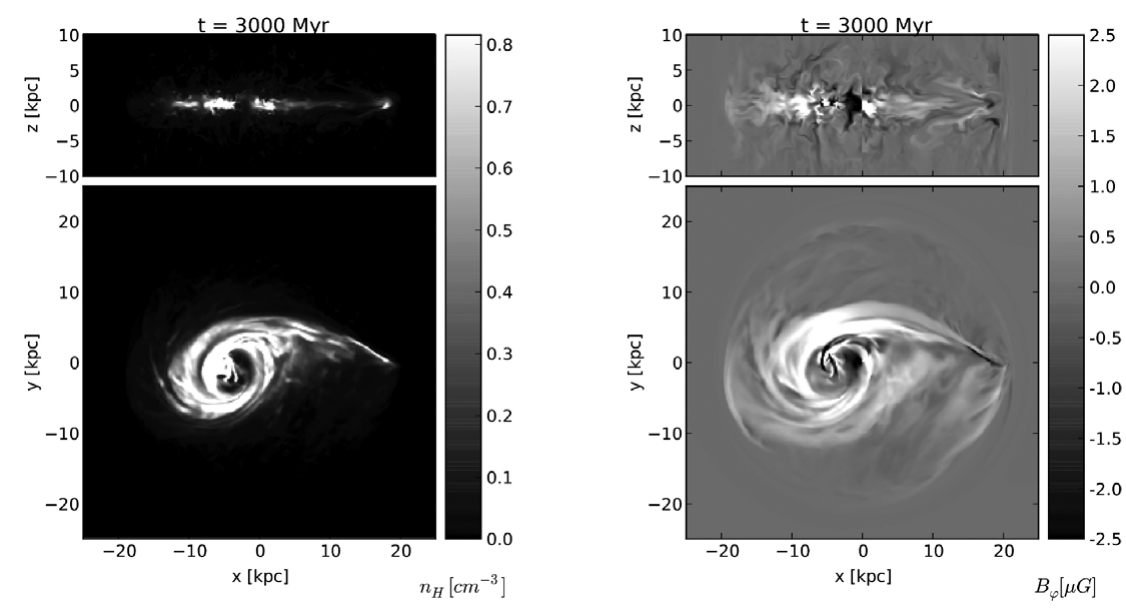

Figure 4. Gas density (left panel) and magnetic field (right panel) corresponding to the gravitational potential of the N-body system at the right plot of Fig.3

\section{Summary and conclusions}

In this paper we have reviewed galactic and intergalactic magnetic field amplification mechanisms. The variety of available models indicates that galactic and intergalactic magnetic fields can be amplified by several different mechanisms, on different timescales, and on different spatial scales. The relatively short amplification timescales of small-scale dynamos point toward the possibility that galactic magnetic fields were first amplified to the equipartition values (corresponding to several $\mu \mathrm{G}$ ) within a few $10^{8} \mathrm{yr}$ (e-folding time of $\sim 50 \mathrm{Myr}$ ). The short amplification timescales, imply that equipartition turbulent magnetic field were present in galaxies already at cosmological redshifts $z \sim 10$. In galaxy clusters the estimated e-folding times of small-scale dynamos are about $0.5 \mathrm{Gyr}$, therefore stronger seed fields are required to explain the present-day $\mu \mathrm{G}$ cluster magnetic fields. Numerical experiments show that galaxy mergers as well as striping of galactic magnetic fields in clusters can serve as source of seed magnetic fields in the intracluster medium.

Regular magnetic fields are generated in disk galaxies by large-scale dynamos on timescales of a few $10^{8} \mathrm{yr}$. Magnetic helicity conservation implies that a net flux of magnetic helicity is necessary for amplification of large-scale magnetic fields in galactic disks. In realistic galactic disk models, supernovae provide the energy necessary to drive galactic winds via expansion of the shock heated gas (Gressel et al., 2008b; Gressel et al., 2008a) and/or buoyancy of the CR (Hanasz et al., 2004; Hanasz et al., 2009a; Hanasz et al., 2009b) component of ISM. The dynamo models powered by supernovae exhibit similarities to the mean field $\alpha \omega$-dynamos. These models plausibly benefit from vertical winds transporting small-scale fields out of the disk. We note that detailed consideration of helicity transport by galactic winds is needed for these dynamo models.

We have shown that the contribution of CRs to the dynamics of the ISM on a global galactic scale leads to a very efficient magnetic field amplification on the timescale of galactic rotation. The model applying a fixed analytical gravitational potential reveals a large scale regular magnetic field with apparent spiral structure in the face-on view and a X-shaped structure in the edge-on view. In the presence of spiral perturbations excited in the stellar component by a companion galaxy, the magnetic field structure follows these perturbations in the stellar and gaseous components. The magnetic field structure becomes less regular compared to the axisymmetric case. Dynamical magnetic field structures with opposite polarities develop within the disk and are present even 
at the saturation phase of the dynamo. Moreover, during the coalescence phase of the two galaxies the magnetic field structure becomes irregular as observed in M51. An important part of the CR-driven dynamo is the galactic wind which reaches velocities of a few hundred $\mathrm{km} / \mathrm{s}$ at galactic altitudes of a few $\mathrm{kpc}$. The mass of gas transported out of the disk is about $1 M_{\odot} /$ yr for the star formation rate of the Milky Way.

\section{Acknowledgements}

This work was partially supported by Polish Ministry of Science and Higher Education through the grant N203 511038. This work is also a part of POWIEW project supported by the European Regional Development Fund in the Innovative Economy Programme (POIG.02.03.00-00-018/08). The computations have been done in TASK Gdańsk Academic Computer Centre and Poznań Networking and Supercomputing Center (PCSS).

\section{References}

Allen, C. \& Santillan, A.: 1991, Revista Mexicana de Astronomia y Astrofisica 22, 255

Arshakian, T. G., Beck, R., Krause, M., \& Sokoloff, D.: 2009, A\&A 494, 21

Balbus, S. A. \& Hawley, J. F.: 1998, Reviews of Modern Physics 70, 1

Beck, A. M., Lesch, H., Dolag, K., Kotarba, H., Geng, A., \& Stasyszyn, F. A.: 2012, MNRAS 422, 2152

Beck, R.: 2012, Space Sci Rev 166, 215

Beck, R., Brandenburg, A., Moss, D., Shukurov, A., \& Sokoloff, D.: 1996, ARAA 34, 155

Bell, E. F.: 2003, ApJ 586, 794

Berezinskii, V. S., Bulanov, S. V., Dogiel, V. A., \& Ptuskin, V. S.: 1990, Astrophysics of cosmic rays, Amsterdam: North-Holland, 1990, edited by Ginzburg, V.L.

Bisnovatyi-Kogan, G. S., Ruzmaikin, A. A., \& Syunyaev, R. A.: 1973, Soviet Astronomy 17, 137

Blackman, E. G. \& Field, G. B.: 2000, ApJ 534, 984

Bonafede, A., Dolag, K., Stasyszyn, F., Murante, G., \& Borgani, S.: 2011, MNRAS 418, 2234

Brandenburg, A. \& Subramanian, K.: 2005, Phys Rep 417, 1

Chyży, K. T.: 2008, A\&A 482, 755

Dolag, K., Bartelmann, M., \& Lesch, H.: 2002, A\&A 387, 383

Donnert, J., Dolag, K., Lesch, H., \& Müller, E.: 2009, MNRAS 392, 1008

Dubois, Y. \& Teyssier, R.: 2010, A\&A 523, A72

Dziourkevitch, N., Elstner, D., \& Rüdiger, G.: 2004, A $6 A$ 423, L29

Enßlin, T., Vogt, C., \& Pfrommer, C.: 2005, in K. T. Chyzy, K. Otmianowska-Mazur, M. Soida, and R.-J. Dettmar (eds.), The Magnetized Plasma in Galaxy Evolution, pp 231-238

Ferriere, K.: 1998, ApJ 497, 759

Fletcher, A., Beck, R., Shukurov, A., Berkhuijsen, E. M., \& Horellou, C.: 2011, MNRAS 412, 2396

Geng, A., Kotarba, H., Bürzle, F., Dolag, K., Stasyszyn, F., Beck, A., \& Nielaba, P.: 2012, MNRAS 419, 3571

Giacalone, J. \& Jokipii, J. R.: 1999, ApJ 520, 204

Gissinger, C., Fromang, S., \& Dormy, E.: 2009, MNRAS 394, L84

Govoni, F. \& Feretti, L.: 2004, International Journal of Modern Physics D 13, 1549

Gressel, O., Elstner, D., \& Rüdiger, G.: 2011, in A. Bonanno, E. de Gouveia Dal Pino,

A. G. \& Kosovichev (eds.), IAU Symposium, Vol. 274 of IAU Symposium, pp 348-354

Gressel, O., Elstner, D., Ziegler, U., \& Rüdiger, G.: 2008a, A\&A 486, L35 
Gressel, O., Ziegler, U., Elstner, D., \& Rüdiger, G.: 2008b, Astronomische Nachrichten 329, 619

Hanasz, M., Kowal, G., Otmianowska-Mazur, K., \& Lesch, H.: 2004, ApJL 605, L33

Hanasz, M. \& Lesch, H.: 2003, A 8 A 412, 331

Hanasz, M., Otmianowska-Mazur, K., Kowal, G., \& Lesch, H.: 2006, Astronomische Nachrichten 327, 469

Hanasz, M., Otmianowska-Mazur, K., Kowal, G., \& Lesch, H.: 2009a, A $\& A$ 498, 335

Hanasz, M., Otmianowska-Mazur, K., \& Lesch, H.: 2002, A\&A 386, 347

Hanasz, M., Wóltański, D., \& Kowalik, K.: 2009b, ApJL 706, L155

Kotarba, H., Lesch, H., Dolag, K., Naab, T., Johansson, P. H., Donnert, J., \& Stasyszyn, F. A.: 2011, MNRAS 415, 3189

Kowal, G., Lazarian, A., Vishniac, E. T., \& Otmianowska-Mazur, K.: 2009, ApJ 700, 63 Kowal, G., Otmianowska-Mazur, K., \& Hanasz, M.: 2006, A\& A 445, 915

Krause, F. \& Raedler, K.-H.: 1980, Mean-field magnetohydrodynamics and dynamo theory Krause, M.: 2009, in Revista Mexicana de Astronomia y Astrofisica Conference Series,

Vol. 36 of Revista Mexicana de Astronomia y Astrofisica, vol. 27, pp 25-29

Kronberg, P. P.: 1994, Reports on Progress in Physics 57, 325

Kulpa-Dybeł, K., Otmianowska-Mazur, K., Kulesza-Żydzik, B., Hanasz, M., Kowal, G., Wóltański, D., \& Kowalik, K.: 2011, ApJL 733, L18

Kulsrud, R. M. \& Zweibel, E. G.: 2008, Reports on Progress in Physics 71(4), 046901

Lesch, H. \& Chiba, M.: 1995, A\&A 297, 305

Mac Low, M.-M. \& Klessen, R. S.: 2004, Reviews of Modern Physics 76, 125

Otmianowska-Mazur, K., Kowal, G., \& Hanasz, M.: 2007, ApJ 668, 110

Otmianowska-Mazur, K., Soida, M., Kulesza-Żydzik, B., Hanasz, M., \& Kowal, G.: 2009, ApJ 693, 1

Otmianowska-Mazur, K. \& Vollmer, B.: 2003, A\&A 402, 879

Parker, E. N.: 1966, ApJ 145, 811

Parker, E. N.: 1992, ApJ 401, 137

Rees, M. J.: 2006, Astronomische Nachrichten 327, 395

Ruzmaikin, A. A., Sokolov, D. D., \& Shukurov, A. M.: 1988, Magnitnye polia galaktik

Schlickeiser, R. \& Lerche, I.: 1985, A\& A 151, 151

Sellwood, J. A. \& Balbus, S. A.: 1999, ApJ 511, 660

Shukurov, A.: 2004, ArXiv Astrophysics e-prints

Shukurov, A., Sokoloff, D., Subramanian, K., \& Brandenburg, A.: 2006, A $\mathscr{E} A$ 448, L33

Siejkowski, H., Soida, M., Otmianowska-Mazur, K., Hanasz, M., \& Bomans, D. J.: 2010, $A \mathscr{G} A$ 510, A97+

Subramanian, K., Shukurov, A., \& Haugen, N. E. L.: 2006, MNRAS 366, 1437

Syrovatskii, S.: 1970, in H. Habing (ed.), Interstellar Gas Dynamics, p. p. 192

Vainshtein, S. I. \& Cattaneo, F.: 1992, ApJ 393, 165

Wang, P. \& Abel, T.: 2009, ApJ 696, 96

Wetzstein, M., Nelson, A. F., Naab, T., \& Burkert, A.: 2009, ApJS 184, 298

Widrow, L. M.: 2002, Reviews of Modern Physics 74, 775

Wóltański, D., Hanasz, M., \& Kowalik, K.: 2013, (in prep) 